\title{
New Performance Indices for 6R Robots
}

\author{
G. Nawratil \\ Vienna University of Technology, Institute of Discrete Mathematics and \\ Geometry, Wiedner Hauptstrasse 8-10/104, Vienna, A-1040, Austria
}

\begin{abstract}
In this paper we introduce four new posture-dependent performance indices for control. Two of them are based on an object-oriented metric in the workspace (endeffector dependent) and the other on a linearized approximation of direct kinematics (end-effector independent). All newly defined indices are invariant under rigid-body motions and similarities, they have a geometric meaning, and they can be computed in real time. We also give a geometric interpretation of the characteristic length.
\end{abstract}

Key words: 6R robot, performance index, distance measure, characteristic length

\section{Introduction}

According to Angeles [1], a performance index of a robotic mechanical system is a scalar quantity that measures how well the system behaves with regard to force and motion transmission. In this paper we introduce four new posture-dependent performance indices for control. Two of them depend on the end-effector EE (EE dependent) and the other two do not (EE independent). The indices defined in chapter 2 and 3 assign to each posture $\mathcal{K}$ of the configuration space a scalar $P I(\mathcal{K})$ which has the following six properties:

1. $P I(\mathcal{K}) \geq 0$ for all $\mathcal{K}$ of the configuration space,

2. $\operatorname{PI}(\mathcal{K})=0$ if and only if $\mathcal{K}$ is singular,

3. $P I(\mathcal{K})$ is invariant under Euclidean motions,

4. $P I(\mathcal{K})$ is invariant under similarites,

5. $P I(\mathcal{K})$ has a geometric meaning,

6. $\operatorname{PI}(\mathcal{K})$ is computable in real time.

Email address: nawratil@geometrie.tuwien.ac.at (G. Nawratil). 
Many papers have been written on this topic. The two best known and most used concepts in this direction are probably the condition number (see section 2.1) and the manipulability (see section 3.1). Both are based on the manipulator's Jacobian

$$
\mathbf{J}=\left(\begin{array}{ccc}
\mathbf{a}_{1} & \ldots & \mathbf{a}_{6} \\
\mathbf{p}_{1} \times \mathbf{a}_{1} & \ldots & \mathbf{p}_{6} \times \mathbf{a}_{6}
\end{array}\right),
$$

where $\mathbf{a}_{i}$ describes the unit vector along the $i^{\text {th }}$ joint axis $\mathbf{r}_{i}$ and $\mathbf{p}_{i}$ the position vector of a point on this axis. All coordinates are measured with regard to a common reference frame with origin $O$. The $i^{\text {th }}$ column of the Jacobian equals the normalized Plücker vector of the arbitrarily directed $i^{\text {th }}$ axis $r_{i}$. In the following we denote the moment vector $\mathbf{p}_{i} \times \mathbf{a}_{i}$ by $\widehat{\mathbf{a}}_{i}$ and we use the notation of dual vectors:

$$
\underline{\mathbf{x}}_{i}:=\mathbf{x}_{i}+\varepsilon \widehat{\mathbf{x}}_{i}=\left(\mathbf{x}_{i}, \widehat{\mathbf{x}}_{i}\right) .
$$

If the joint variables $\theta_{1}, \ldots, \theta_{6}$ are functions of time $t$, a constrained motion of the EE system $\Sigma_{6}$ against the fixed system $\Sigma_{0}$ is determined. Because of the spatial Three-Pole-Theorem (see [8]) the one-parametric motion $\Sigma_{6} / \Sigma_{0}$ can be composed from the relative motions $\Sigma_{1} / \Sigma_{0}, \ldots, \Sigma_{6} / \Sigma_{5}$ and therefore the instantaneous twist $\underline{\mathbf{q}}_{6 \mid 0}=\left(\mathbf{q}_{6 \mid 0}, \widehat{\mathbf{q}}_{6 \mid 0}\right)$ is computable as

$$
\underline{\mathbf{q}}_{6 \mid 0}=\mathbf{J} \boldsymbol{\omega} \quad \text { with } \quad \boldsymbol{\omega}=\left(\omega_{1 \mid 0}, \ldots, \omega_{6 \mid 5}\right)^{T} \quad \text { and } \quad \omega_{i \mid i-1}=\frac{d \theta_{i}}{d t} .
$$

The rank of $\mathbf{J}$ gives the number of instantaneous degrees of freedom (dof). If the rank drops below 6 , then there exists at least one angular velocity ratio $\mathbb{R} \boldsymbol{\omega} \neq \mathbf{o}$ such that the EE has an instantaneous standstill while the joints are still moving. The columns in $\mathbf{J}$ are linearly dependent. Therefore we can characterize the singular postures of $6 \mathrm{R}$ robots as follows:

Theorem 1 A posture of a non-redundant 6-dof manipulator is singular if and only if the determinant of the Jacobian vanishes. Exactly in this case the axes belong to a linear line complex.

\section{EE dependent performance indices}

\subsection{Review: Condition Number CDN}

According to Salisbury and Craig [7], the condition number is the positive square root of the ratio of the maximal and minimal eigenvalue of $\mathbf{C}=\mathbf{J}^{T} \mathbf{J}$. $C D N^{-1}$ lies in the interval $[0,1]$ and therefore this enjoys the properties 1 and 2 . It should be noted that postures with the maximum value 1 are called 
isotropic. $C D N^{-1}$ is not invariant under Euclidean motions with the exeption of rotations of the reference frame about $O$. But the real problem, which causes the variance of $C D N^{-1}$ under similarities, occurs from the dimensional inhomogeneity of $\mathbf{J}$. To overcome this deficiencies, different methods were introduced. In the following we discuss the two most used concepts:

\subsubsection{Concept $A$}

To achieve the invariance under Euclidean motions some authors (e.g. [1,2]) link $O$ with an EE point, namely the operation point $O P$. The characteristic length $C L$ introduced by Tandirci et al. [9] is used to overcome the problem of inhomegeneity. The last three rows of $\mathbf{J}$ that have units of length are devided by $C L$ which means that the matrix $\mathbf{C}$ defined above now reads

$$
\mathbf{C}=\mathbf{J}^{T} \mathbf{L J} \quad \text { with } \quad \mathbf{L}=\left(\begin{array}{cc}
\mathbf{I}_{3} & \mathbf{O}_{3} \\
\mathbf{O}_{3} & \frac{\mathbf{I}_{3}}{C L^{2}}
\end{array}\right) .
$$

where $\mathbf{I}_{i}$ is the $i \times i$ identity matrix and $\mathbf{O}_{i}$ the $i \times i$ zero matrix. The condition number based on this concept is denoted by $C D N_{C L}$. Due to the definition of $C L$ as the solution of an optimization problem (see [9]), a meaningful geometric interpretaion of $C L$ and therefore also of $C D N_{C L}$ is still missing. As by-product of our considerations, we will present a geometric interpretation of $C L$, which puts its original definition into question.

\subsubsection{Concept B}

Gosselin [3] solved the problem by describing the global velocity of the EE by the velocity of three non-collinear points. In this way the desired quality was achieved. The drawback of this concept is that the choice of the reference points is arbitrary and that the index is not invariant under changes of these points. Angeles and Lopez-Cajun also suggested in [2] to use not only three points but the vertices of a platonic solid centered in $O P$. But it is still an open question which platonic solid with which radius should be taken because the index is not invariant under this choice. We will answer these question by defining $C D N_{O E}$ in section 2.4.

\subsection{Object-oriented metric and the operation ellipsoid}

In order to provide a geometric interpretation and avoid a indeterminacy of number and choice of reference points of the newly defined performance indices, we use a geometrically meaningful metric in the space of rigid body 
motions. The used metric is motivated by the following consideration:

Basically, one is not interested in the manipulation of a single point $(O P)$ or of three points, but in that of a complete object $\mathcal{O}$, which can, e.g., be a welding tool or a load to be transported. Therefore we need an object-oriented metric which allows us to define a distance between any two poses of $\mathcal{O}$.

The metric used here was introduced by Hofer et al. [4] and has its origin in the registration problem with known correspondences. We represent the object of interest by a finite number of points $\mathrm{P}_{i}(i=1, . ., N)$. By means of this point cloud it is possible to define the distance between any two poses $\mathcal{O}(0)$ and $\mathcal{O}(t)$ as follows:

$$
d(\mathcal{O}(0), \mathcal{O}(t))^{2}:=\sum_{i=1}^{N}\left\|\mathbf{p}_{i}(0)-\mathbf{p}_{i}(t)\right\|^{2}
$$

where $\mathbf{p}_{i}$ denotes the position vector of $\mathrm{P}_{i}$. This metric only depends on the centroid $\mathbf{b}_{\mathcal{O}}$ and the the covariance matrix $\mathbf{D}_{\mathcal{O}}$ with respect to the centroid $\mathbf{b}_{\mathcal{O}}$ given by:

$$
\mathbf{b}_{\mathcal{O}}:=N^{-1} \sum_{i=1}^{N} \mathbf{p}_{i} \quad \text { and } \quad \mathbf{D}_{\mathcal{O}}:=\sum_{i=1}^{N}\left(\mathbf{p}_{i}-\mathbf{b}_{\mathcal{O}}\right)\left(\mathbf{p}_{i}-\mathbf{b}_{\mathcal{O}}\right)^{T}
$$

Therefore we can replace the points $\mathrm{P}_{i}(i=1, . ., N)$ by the six special points $\mathrm{S}_{i}$ with

$$
\mathbf{s}_{i}:=\mathbf{b}_{\mathcal{O}}+\sqrt{\frac{\lambda_{i}}{2}} \mathbf{e}_{i}, \quad \mathbf{s}_{i+3}:=\mathbf{b}_{\mathcal{O}}-\sqrt{\frac{\lambda_{i}}{2}} \mathbf{e}_{i} \quad i=1,2,3
$$

where $\lambda_{i}$ denotes any eigenvalue and $\mathbf{e}_{i}$ a corresponding unit eigenvector of $\mathbf{D}_{\mathcal{O}}$. This does not change the centroid, the covariance matrix and the inertia tensor $\mathbf{T}_{\mathcal{O}}$ of $\mathcal{O}$, which is given by:

$$
\mathbf{T}_{\mathcal{O}}:=\operatorname{tr}\left(\mathbf{D}_{\mathcal{O}}\right) \mathbf{I}_{3}-\mathbf{D}_{\mathcal{O}}
$$

Due to this fact we can expand the operation point to the more practical concept of an operation ellipsoid as follows:

Definition 1 The operation ellipsoid $O E$ denotes that part of the end-effector bounded by an ellipsoid $\mathcal{E}$ with $\operatorname{Vol}(\mathcal{E}) \neq 0$, which one intends to manipulate. The $O E \in \Sigma_{6}$ is determined by the six vertices $\boldsymbol{S}_{i}$ of $\mathcal{E}$. 


\subsection{The Performance Index $P I_{O E}$}

$O E$ is given by its vertices $\mathrm{S}_{i}$ with $\mathbf{s}_{i}:=\left(s_{1}^{i}, s_{2}^{i}, s_{3}^{i}\right)$ for $i=1, . ., 6$. Due to (4) the distance between any two poses $O E(0)$ and $O E(t)$ can be written as:

$$
d(O E(0), O E(t))^{2}=\sum_{i=1}^{6}\left\|\mathbf{s}_{i}(0)-\mathbf{s}_{i}(t)\right\|^{2}
$$

The first order Taylor approximation of the posture $O E(t)$ yields:

$$
d(O E(0), O E(t))^{2}=\sum_{i=1}^{6}\left\|\mathbf{s}_{i}(0)-\mathbf{s}_{i}(t)\right\|^{2} \approx \sum_{i=1}^{6}\left\|\mathbf{v}\left(\mathrm{S}_{i}\right)\right\|^{2}
$$

where $\mathbf{v}\left(S_{i}\right)$ denotes the velocity vector of the vertex $S_{i} \in \Sigma_{6}$. We are interested in the angular volocity ratio $\mathbb{R} \boldsymbol{\omega}$ which has the lowest effects on the instantaneous displacement of $O E$, i.e., which causes the worst motion transmission with respect to $O E$. This ratio can be computed as follows:

We search for the minimum of the objective function

$$
\zeta(\boldsymbol{\omega}): \quad \sum_{i=1}^{6}\left\|\mathbf{v}\left(\mathrm{S}_{i}\right)\right\|^{2} \quad \text { with } \quad \mathbf{v}\left(\mathrm{S}_{i}\right)=\sum_{j=1}^{6} \widehat{\mathbf{a}}_{j} \omega_{j \mid j-1}+\left(\mathbf{a}_{j} \omega_{j \mid j-1} \times \mathbf{s}_{i}\right)
$$

under the side condition (= normalization condition)

$$
\nu(\boldsymbol{\omega}): \quad \boldsymbol{\omega}^{T} \boldsymbol{\omega}=\boldsymbol{\omega}^{T} \mathbf{I}_{6} \boldsymbol{\omega}=1
$$

The function $\zeta(\boldsymbol{\omega})$ is a quadratic form in the variables $\omega_{i \mid i-1}$, and so we can rewrite it as $\boldsymbol{\omega}^{T} \mathbf{Z} \boldsymbol{\omega}$ with $\mathbf{Z}=\mathbf{J}^{T} \mathbf{W J}$, where $\mathbf{W}$ is given by

$$
\mathbf{W}=\sum_{i=1}^{6}\left(\begin{array}{cc}
\mathbf{S}_{i}^{T} \mathbf{S}_{i} & \mathbf{S}_{i}^{T} \\
\mathbf{S}_{i} & \mathbf{E}_{3}
\end{array}\right) \quad \text { with } \quad \mathbf{S}_{i}=\left(\begin{array}{rrr}
0 & s_{3}^{i}-s_{2}^{i} \\
-s_{3}^{i} & 0 & s_{1}^{i} \\
s_{2}^{i}-s_{1}^{i} & 0
\end{array}\right)
$$

We solve the minimization problem (10) by introducing a Lagrange multiplier $\lambda$. With $\nabla \zeta=2 \mathbf{Z} \boldsymbol{\omega}$ and $\nabla \nu=2 \mathbf{I}_{6} \boldsymbol{\omega}$, minimization results in the eigenvalue problem $\left(\mathbf{Z}-\lambda \mathbf{I}_{6}\right) \boldsymbol{\omega}=\mathbf{o}$. This system of linear equations has a nontrivial solution, if and only if the determinant of $\left(\mathbf{Z}-\lambda \mathbf{I}_{6}\right)$ vanishes. Every eigenvalue $\lambda_{i}$ of $\mathbf{Z}$ is linked with an eigenvector $\boldsymbol{\omega}_{i}$. Because of

$$
\zeta\left(\boldsymbol{\omega}_{i}\right)=\boldsymbol{\omega}_{i}^{T} \mathbf{Z} \boldsymbol{\omega}_{i}=\lambda_{i} \boldsymbol{\omega}_{i}^{T} \mathbf{I}_{6} \boldsymbol{\omega}_{i}=\lambda_{i}
$$

the smallest eigenvalue $\lambda_{\min }$ corresponds to the required solution. 


\subsubsection{Definition of $P I_{O E}$}

As $O E$ is rigidly attached $\Sigma_{6}$ the relative pose between $O E$ and the $6^{\text {th }}$ joint axis $r_{6}$ is constant. Hence the rotation of $O E$ about $r_{6}$ can be done in any configuration with the same performance $D C$ defined as

$$
D C:=\zeta(0, . ., 0,1)=\sum_{i=1}^{6}{\overline{r_{6}}}_{i}^{2} \geq \lambda_{\min } \quad D C=\text { const. } \forall \mathcal{K}
$$

where $\overline{r_{6} S_{i}}$ denotes the distance of $S_{i}$ from $r_{6}$. Therefore $D C$ is an design constant of the $6 \mathrm{R}$ robot.

Definition 2 The EE dependent performance index $P I_{O E}(\mathcal{K})$ of the configuration $\mathcal{K}$ with respect to the operation ellipsoid $O E$ (Def. 1) is defined as

$$
P I_{O E}(\mathcal{K}):=+\sqrt{\frac{\lambda_{\min }}{D C}} \quad \text { with } \quad P I_{O E}(\mathcal{K}) \in[0,1]
$$

where $\lambda_{\text {min }}$ denotes the smallest eigenvalue of $\mathbf{Z}=\mathbf{J}^{T} \mathbf{W} \mathbf{J}$ and $D C$ as in (14).

Theorem $2 P I_{O E}(\mathcal{K})$ is well defined.

Proof: In the non-singular case of the robot, $\mathbf{Z}$ is positive definite because $\zeta(\boldsymbol{\omega})$ depends only on the distances of $\mathrm{S}_{i}$ from the instantaneous screw axis $\mathrm{q}$ and on the angular velocity $\omega_{6 \mid 0}$ and the translatory velocity $\widehat{\omega}_{6 \mid 0}$ of screw $\underline{\mathbf{q}}_{6 \mid 0}$ according to

$$
\sum_{i=1}^{6}\left\|\mathbf{v}\left(\mathrm{S}_{i}\right)\right\|^{2}=\omega_{6 \mid 0}^{2} \sum_{i=1}^{6} \overline{\mathrm{qS}}_{i}^{2}+6 \widehat{\omega}_{6 \mid 0}^{2} .
$$

$\mathbf{Z}$ would be positive semidefinite in a regular configuration if and only if $O E$ would degenerate to a single line segment or a point, which is not possible because of Def. 1 . On the other hand $\mathbf{Z}$ is positive semidefinite in a singular case because of $\mathbf{Z}=\mathbf{J}^{T} \mathbf{W} \mathbf{J}$.

It must also be proved that the index is invariant under the choice of the vertices of $O E$ if they are not determined uniquely. This is the case when $O E$ is a sphere or an ellipsoid of revolution. Due to (16) we only have to prove that the sum of squares of the distances of $S_{i}$ from $q$ is invariant under the choice of the vertices $S_{i}$. But this is trivially true.

Theorem $3 P I_{O E}(\mathcal{K})$ has all six properties required in section 1.

Proof: Due to Theorem 2, $P I_{O E}(\mathcal{K})$ enjoys the properties 1 and 2. $P I_{O E}(\mathcal{K})$ is also invariant under similarities because it was defined as a ratio. This index satisfies also the $3^{r d}$ point because the vertices $S_{i}$ of $O E$ are rigidly attached $\Sigma_{6}$. The $5^{\text {th }}$ property is trivially true because $P I_{O E}(\mathcal{K})$ is based on a geometrically meanigful metric with respect to $O E$. $P I_{O E}(\mathcal{K})$ is computable in real time, because one only has to calculate the eigenvalues of a $6 \times 6$ matrix. 


\subsection{The Performance Index $C D N_{O E}$}

The second new EE dependent performance index introduced in this paper is the condition number based on the object oriented metric and the $O E$. This index is denoted by $C D N_{O E}$ and defined as follows:

Definition 3 The EE dependent performance index $C D N_{O E}(\mathcal{K})$ of the configuration $\mathcal{K}$ with respect to the operation ellipsoid $O E$ (Def. 1) is defined as

$$
C D N_{O E}(\mathcal{K}):=+\sqrt{\frac{\lambda_{\max }}{\lambda_{\min }}} \quad \text { with } \quad C D N_{O E}^{-1}(\mathcal{K}) \in[0,1]
$$

where $\lambda_{\min }$ resp. $\lambda_{\max }$ is the smallest resp. largest eigenvalue of $\mathbf{Z}=\mathbf{J}^{T} \mathbf{W J}$.

Theorem $4 C D N_{O E}(\mathcal{K})$ is well defined and has all six properties required in section 1.

Proof: The proof follows immediately from Theorem 2 and Theorem 3.

\subsubsection{Geometric interpretation of the characteristic length $C L$}

With the help of $C D N_{O E}(\mathcal{K})$ we are able to give a geometric interpretation of $C L$. We assume that $O E$ is centered in $O P$ and that the axes of the reference frame are the principal axes of $O E$. Therefore $S_{i}$ have the following coordinates:

$$
\mathbf{s}_{1,4}=( \pm a, 0,0) \quad \mathbf{s}_{2,5}=(0, \pm b, 0) \quad \mathbf{s}_{3,6}=(0,0, \pm c) .
$$

The matrix $\mathbf{W}$ defined in equation (12) simplifies to

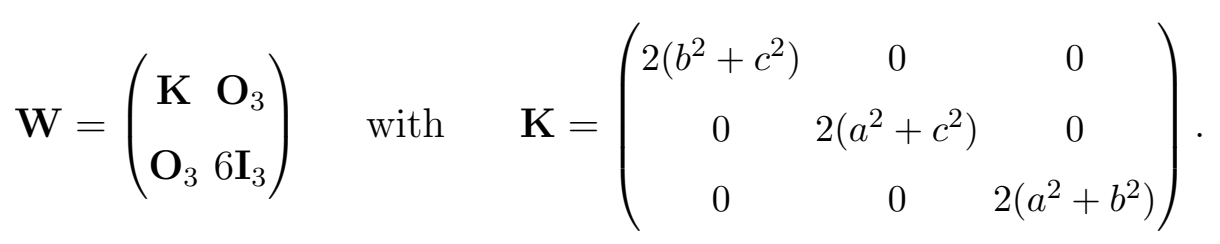

Theorem 5 The characteristic length CL of $C D N_{C L}(\mathcal{K})$ equals $R \sqrt{2 / 3}$ where $R$ is the radius of that operation sphere centered in the operation point, which causes a minimal $C D N_{O E}(\mathcal{K})$ over the manipulators configuration space.

Proof: The indices $C D N_{O E}(\mathcal{K})$ and $C D N_{C L}(\mathcal{K})$ are equal if and only if $\mathbf{W}=$ $6 C L^{2} \mathbf{L}$. This system of linear equations is fulfilled, if $O E$ becomes an operation sphere with radius $R:=a=b=c=C L \sqrt{3 / 2}$.

In view of Theorem 5 the $C D N_{C L}$ can be seen as a special case of the $C D N_{O E}$. This geometric interpretation of $C L$ as the radius of an operation sphere, puts the original definition of $C L$ for the following reasons into question: 
- Robot Design According to Angeles and Lopez-Cajun [2] a robot has an optimal architecture, if there exists a $C L$ and a configuration $\mathcal{K}_{i}$ within the workspace, such that $C D N_{C L}\left(\mathcal{K}_{i}\right)=1$. But until now the value of $C L$ was unimportant, because it was meaningless. Due to the interpretation of $C L$ given in Theorem 5 the characteristic length should not be the result of an optimization procedure but a design constant, which must be predefined. When designing a robot, one has to determine first an operation sphere and therefore a $C L$. After this the design of the robot should be optimized in such a way that there exists an isotropic configuration within the workspace. It should be said that it is not possible to achieve an isotropic configuration if $O P$ lies on the $6^{\text {th }}$ axis. ${ }^{1}$

- Robot Control The operation sphere with radius $C L \sqrt{3 / 2}$ generally does not fit the object of interest. Therefore the $C D N_{C L}(\mathcal{K})$ is no adequate index for robot control. We suggest to take $P I_{O E}(\mathcal{K})$ or $C D N_{O E}(\mathcal{K})$.

We suggest the following redefinition of the characteristic length, which is based on Theorem 5:

Definition 4 The characteristic length is defined as $C L:=R \sqrt{2 / 3}$, where $R$ is the radius of the operation sphere of Def. 1. The center of the sphere is defined as the operation point.

Remark: We can replace the matrix $\mathbf{W}$ of (19) by $\frac{1}{6} \mathbf{W}$ without changing $C D N_{O E}$. Then the lower right $3 \times 3$ matrix is the identity matrix and the upper left $3 \times 3$ matrix $\left(=\frac{1}{6} \mathbf{K}\right)$ equals the inertia tensor of the operation ellipsoid materialized as an ellipsoidal shell $\mathcal{E}$ of mass 1 . Therefore $C D N_{O E}$ can also be interpreted as the square root of the ratio of the minimal and maximal kinetic energy of $\mathcal{E}$ under the side condition $\nu(\boldsymbol{\omega})$ of (11). Thus $P I_{O E}$ of Def. 2 can be seen as the square root of the ratio of the minimal kinetic energy of $\mathcal{E}$ under the side conition $\nu(\boldsymbol{\omega})$ and the rotational energy of $\mathcal{E}$ with respect to the rotation about $r_{6}$ with angular velocity 1 .

An adapted version of $C D N_{O E}$ can be used as an EE dependent performance index for Stewart Gough Platforms (see [6]). A new EE independent performance index for these manipulators has been presented in [5].

\footnotetext{
1 A necessary condition for achieving a isotropic configuration is that $O P$ has the same distance from all joint axes $\mathrm{r}_{i}$, for $i=1, \ldots, 6$.
} 


\section{$3 \quad$ EE independent performance index}

\subsection{Review: Manipulability $M P B$}

This performance index introduced by Yoshikawa [10] is not invariant under similarities, because for non-redundant manipulators it equals nothing else but $|\operatorname{det}(\mathbf{J})|$. This index obviously enjoys properties 1 and 2 , but also property 3 : Let $\mathbf{R}$ be an orthogonal matrix which induces a rotation $\rho$ of the reference frame and let $\mathbf{t}:=(x, y, z)^{T}$ be the vector of any translation $\tau$. We only have to prove that the determinant of $\mathbf{J}$ is invariant under translations and rotations. Plücker coordinates transform as follows:

$$
(\mathbf{a}, \widehat{\mathbf{a}}) \stackrel{\rho}{\longrightarrow}(\mathbf{R} \mathbf{a}, \mathbf{R} \widehat{\mathbf{a}}) \quad \text { and } \quad(\mathbf{a}, \widehat{\mathbf{a}}) \stackrel{\tau}{\longrightarrow}(\mathbf{a}, \widehat{\mathbf{a}}+(\mathbf{t} \times \mathbf{a}))
$$

Therefore the invariance of $|\operatorname{det}(\mathbf{J})|$ immediately follows from $\operatorname{det}(\mathbf{R})=1$.

In the vector space $\mathbb{R}^{6}$ of instantaneous screws the subset of all $\underline{\mathbf{q}}_{6 \mid 0}$ with $\left|\omega_{i \mid i-1}\right| \leq 1$ for $i=1, \ldots, 6$, is a parallelepiped by (2), which has the volume $2^{6} \cdot|\operatorname{det}(\mathbf{J})|$. Therefore the given index is proportional to this volume. But this volume neither gives us information about the capability to manipulate the EE because $M P B$ is EE independent. Nor it has a geometrically meaning for the manipulator, due to the following reason: $M P B$ does not take the geometry of the manipulator into consideration, because exchanging columns of $\mathbf{J}$ results in exchanging the order of the joint axes, which has no influence on $|\operatorname{det}(\mathbf{J})|$. Therefore we want to define an EE independent performance index as the distance of the actual posture to the closest singular one, whereas this distance should have a geometric meaning for the manipulator.

\subsection{Preliminary considerations for a distance measure}

It does not make sense to use a purely line geometrical point of view by paying only attention to the actual postures of the six joint axes. And exactly this is the case if one considers only $\mathbf{J}$. Of course, it is always possible to find singular postures close to a given configuration. Let's assume that $\mathcal{C}$ is the linear complex spanned by the first five axes. All lines of $\mathcal{C}$ intersecting the sixth axis $r_{6}$ form a hyperbolic linear congruence if $\mathcal{C}$ is not singular. Replacing $r_{6}$ by an arbitrary line I of this congruence gives a singular configuration. But which $I$ is closest to $r_{6}$ ? How should a distance between the given posture and a close singular one be defined such that we can distinguish between better and worse configurations? Of course, it is obvious using distances and angles of $r_{6}$ to the second axis of the hyperbolic line congruence, but which function of 
angle and distance gives a meaningful positive measure, and which is invariant under scaling and works in all cases?

Therefore, a reasonable way to come up with a geometrically explicable distance measure is to take the possible variability of the joint axes into account. For a serial robot with fixed $r_{1}$ the second axis admits only a one-parametric movement against the frame, and its position influences that of the axes $r_{3}$, $r_{4}$, etc.

Assume that the robot is abruptly stopped when it moves through a certain configuration $\mathcal{K}$ with the instantaneous angular velocity vector $\boldsymbol{\omega}$. Then it overdrives the posture $\mathcal{K}$, whereas each axis $r_{i+1}$ moves according to the instantaneous screw $\underline{\mathbf{q}}_{i \mid 0}$ of the motion of $\Sigma_{i} / \Sigma_{0}$ with

$$
\underline{\mathbf{q}}_{i \mid 0}=\left(\mathbf{q}_{i \mid 0}, \widehat{\mathbf{q}}_{i \mid 0}\right)=\sum_{j=1}^{i} \underline{\mathbf{a}}_{j} \omega_{j \mid j-1} \quad \text { for } \quad i=1, . ., 5 .
$$

Now let for $i=1, \ldots, 5$ the instantaneous rotation about $\mathbf{r}_{i}$ with velocity $\omega_{i \mid i-1}$ operate for a certain time $\delta_{i} \in \mathbb{R}^{+}$. Then the finite rotation about $\mathbf{r}_{i}$ through the angle $\Delta_{i}=\delta_{i} \omega_{i \mid i-1}$ is performed. How must the rotation angles be chosen to reach the closest singularity? Since a rotation of the robot about the first axis $r_{1}$ has no influence on the distance from the closest singular posture, we may assume $\omega_{1 \mid 0}=0$. Depending on the minimization condition which the angular velocities have to fulfill, we will get two different distance measures $D M_{\infty}(\mathcal{K})$ (see section 3.4.1) and $D M_{2}(\mathcal{K})$ (see section 3.4.2) for serial robots between the actual posture $\mathcal{K}$ and the closest singularity.

\subsection{Linearized approximation of direct kinematics}

We start from the posture $\mathcal{K}\left(\theta_{1}, \ldots, \theta_{6}\right)=: \mathcal{K}(\theta)$ of the configuration space, and look for a singular configuration $\mathcal{K}\left(\theta_{1}, \theta_{2}+\Delta_{2}, \ldots, \theta_{5}+\Delta_{5}, \theta_{6}\right)=: \mathcal{K}(\delta)$, where $\Delta_{i}$ should be kept as small as possible. Our two measures will be based on a linearized approximation of this problem in order to reduce the computational costs. This simplification is sufficient for the definition of a distance measure for the following reasons: On the one hand, the approximation is sufficiently close to a singularity. On the other hand, the exact value is not interesting as long as it is larger than a critical distance, which must be predefined. ${ }^{2}$ Then the robot is not in an imminent closeness to a singular posture.

In order to compute the closest singularity we need an approximation of the axes $r_{3}, \ldots, r_{6}$, because the singularity is only determined by the postures of

$\overline{2}$ Experiments will show which value of $D M_{\infty}(\mathcal{K})$ or $D M_{2}(\mathcal{K})$ for given robots can be seen as a sufficient distance from the next singularity. 
the joint axes. As $\underline{\mathbf{a}}_{i}(\delta)$ depends on the angles $\theta_{j}+\Delta_{j}, j=2, \ldots, i-1$, we obtain, by Taylor's formula,

$$
\underline{\mathbf{a}}_{i}(\delta) \approx \underline{\mathbf{a}}_{i}\left(\theta_{2}, \ldots, \theta_{i-1}\right)+\sum_{j=2}^{i-1} \Delta_{j} \frac{\partial \underline{\mathbf{a}}_{i}\left(\theta_{2}, \ldots, \theta_{i-1}\right)}{\partial \theta_{j}}=: \underline{\mathbf{b}}_{i}(\delta),
$$

where $\partial \underline{\mathbf{a}}_{i}(\theta) / \partial \theta_{j}$ expresses the instantaneous change of $\mathbf{r}_{i}$ under the rotation about $r_{j}$ with angular velocity $\omega_{j \mid j-1}=1$. The screw associated to this rotation is $\underline{\mathbf{a}}_{j}$, which implies that

$$
\frac{\partial \underline{\mathbf{a}}_{i}(\theta)}{\partial \theta_{j}}=\underline{\mathbf{a}}_{j}(\theta) \times \underline{\mathbf{a}}_{i}(\theta)
$$

Therefore we get:

$$
\underline{\mathbf{b}}_{i}(\delta)=\underline{\mathbf{a}}_{i}\left(\theta_{2}, \ldots, \theta_{i-1}\right)+\sum_{j=2}^{i-1} \Delta_{j}\left(\underline{\mathbf{a}}_{j} \times \underline{\mathbf{a}}_{i}\right) \quad \text { with }
$$

the primal part

$$
\mathbf{b}_{i}(\delta)=\mathbf{a}_{i}(\theta)+\sum_{j=2}^{i-1} \Delta_{j}\left(\mathbf{a}_{j} \times \mathbf{a}_{i}\right) \quad \text { and }
$$

the dual part

$$
\widehat{\mathbf{b}}_{i}(\delta)=\widehat{\mathbf{a}}_{i}(\theta)+\sum_{j=2}^{i-1} \Delta_{j}\left[\left(\widehat{\mathbf{a}}_{j} \times \mathbf{a}_{i}\right)+\left(\mathbf{a}_{j} \times \widehat{\mathbf{a}}_{i}\right)\right] .
$$

Note that the approximation $\underline{\mathbf{b}}_{i}(\delta)$ of $\underline{\mathbf{a}}_{i}(\delta)$, in general, satisfies neither the Plücker condition $\mathbf{b}_{i}(\delta) \cdot \widehat{\mathbf{b}}_{i}(\delta)=0$ nor the normalization condition $\underline{\mathbf{b}}_{i}(\delta) \cdot \underline{\mathbf{b}}_{i}(\delta)=$ 1. But for small $\Delta_{i}$, we approximately meet these conditions:

$$
\underline{\mathbf{b}}_{i}(\delta) \cdot \underline{\mathbf{b}}_{i}(\delta)=\underline{\mathbf{a}}_{i} \cdot \underline{\mathbf{a}}_{i}+\sum_{j=2}^{i-1} \Delta_{j} \frac{\partial\left(\underline{\mathbf{a}}_{i} \cdot \underline{\mathbf{a}}_{i}\right)}{\partial \theta_{j}}+\frac{1}{2} \sum_{j, k=2}^{i-1} \Delta_{j} \Delta_{k} \frac{\partial^{2}\left(\underline{\mathbf{a}}_{i} \cdot \underline{\mathbf{a}}_{i}\right)}{\partial \theta_{j} \partial \theta_{k}} \approx 1
$$

because the partial derivative of $\underline{\mathbf{a}}_{i} \cdot \underline{\mathbf{a}}_{i}=1$ yields $\frac{\partial\left(\underline{\mathbf{a}}_{i} \cdot \underline{\mathbf{a}}_{i}\right)}{\partial \theta_{j}}=2 \underline{\mathbf{a}}_{i} \frac{\partial \underline{\mathbf{a}}_{i}}{\partial \theta_{j}}=0$.

Therefore the approximation of the determinant of $\mathbf{J}(\delta)$ can be written as:

$$
\begin{array}{r}
\operatorname{det}[\mathbf{J}(\delta)]=\operatorname{det}\left[\underline{\mathbf{a}}_{1}, \underline{\mathbf{a}}_{2}, \underline{\mathbf{a}}_{3}\left(\theta_{2}+\Delta_{2}\right), \ldots, \underline{\mathbf{a}}_{6}\left(\theta_{2}+\Delta_{2}, \ldots, \theta_{5}+\Delta_{5}\right)\right] \approx \ldots \\
\approx \operatorname{det}[\mathbf{J}(\theta)]+\sum_{j=2}^{5} \frac{\partial \operatorname{det}(\mathbf{J})}{\partial \theta_{j}} \Delta_{j} \quad \text { with } \quad \frac{\partial \operatorname{det}(\mathbf{J})}{\partial \theta_{j}}=\sum_{k=j+1}^{6} \operatorname{det}\left(\underline{\mathbf{a}}_{1}, . ., \frac{\partial \underline{\mathbf{a}}_{k}}{\partial \theta_{j}}, . .\right) .
\end{array}
$$

We set $\operatorname{det}[\mathbf{J}(\delta)]=0$ in order to reach a close singularity, and rewrite this equation in the form

$$
\operatorname{det}(\mathbf{J})+\sum_{i=2}^{5} \Delta_{i} \sum_{j=i+1}^{6} \operatorname{det}\left(\mathbf{J}_{i, j}\right)=0
$$

where $\mathbf{J}_{i, j}$ denotes the matrix, where the $j^{\text {th }}$ column of $\mathbf{J}$ is replaced by

$$
\underline{\mathbf{a}}_{i} \times \underline{\mathbf{a}}_{j}=\left(\mathbf{a}_{i} \times \mathbf{a}_{j}, \widehat{\mathbf{a}}_{i} \times \mathbf{a}_{j}+\mathbf{a}_{i} \times \widehat{\mathbf{a}}_{j}\right) .
$$




\subsection{The distance measures $D M_{\infty}$ and $D M_{2}$}

All quadruples $\left(\Delta_{2}, \ldots, \Delta_{5}\right)$, which solve the inhomogenous linear equation (24), are located in a hyperplane $\mathcal{H}$ of the vector space $\mathbb{V}_{\mathcal{K}}$ spanned by $\theta_{2}, \ldots, \theta_{5}$. The variables $\Delta_{i}$ serve as local coordinates in $\mathbb{V}_{\mathcal{K}}$ with origin $\mathcal{K}_{o}:=$ $\mathcal{K}(\theta)$. The zero vector is a solution of (24), if and only if $\mathcal{H}$ passes through $\mathcal{K}_{o}$, which means $\operatorname{det}(\mathbf{J})=0$ (see figure 1 ).

\subsubsection{Definition of $D M_{\infty}$}

We solve equation (24) under the side condition that $\left|\Delta_{i}\right| \leq \Delta_{\mathrm{L}}$ for $i=2, \ldots, 5$, and that $\Delta_{\mathrm{L}}$ is minimal. We get the required quadruple $\left(\Delta_{2}, \ldots, \Delta_{5}\right)$ as the nonempty intersection of $\mathcal{H}$ and the smallest hypercube centered in the origin $\mathcal{K}_{o}$ and with edges parallel to the axes. This nonempty set always contains a vertex $L$ of the hypercube (see figure 1 ). The position vector $\mathbf{l}=\left(l_{2}, \ldots, l_{5}\right)$ of $\mathrm{L}$ with respect to the local coordinate system is given by

$$
l_{i}=\operatorname{sgn}\left(\operatorname{det}(\mathbf{J}) \cdot \frac{\partial \operatorname{det}(\mathbf{J})}{\partial \theta_{i}}\right) \Delta_{\mathrm{L}} \quad \text { with } \quad \Delta_{\mathrm{L}}=\frac{|\operatorname{det}(\mathbf{J})|}{\sum_{j=2}^{5}\left|\frac{\partial \operatorname{det}(\mathbf{J})}{\partial \theta_{j}}\right|} .
$$

Theorem $6 D M_{\infty}(\mathcal{K})$ approximately equals the smallest angle $\Delta_{\mathrm{L}}(26)$ through which one has to rotate simultaneously all joints $\Sigma_{j+1}$ about $\mathbf{r}_{j}(j=2, \ldots, 5)$ with the orientation $\operatorname{sgn}\left(l_{j}\right)$ to pass through a singular posture.

It can happen that the smallest nonempty intersection of the hypercube and $\mathcal{K}$ is one-, two- or three-dimensional, because $\mathcal{H}$ can touch the hypercube along an edge, a plane or a hyperplane. These exeptional cases manifest themselves in the above formulas as follows:

Depending on the dimension of the intersection some $l_{i}$ 's vanish because the $i^{\text {th }}$ coordinate of the normal vector of $\mathcal{H}$ equals zero. So the distance from the singularity does not depend on the respective $\Delta_{i}$ 's.

\subsubsection{Definition of $D M_{2}$}

As an alternative, we optimize equation (24) such that the sum of the squares of the angles is minimal. That means, that we are searching for the point $\mathrm{Q} \in \mathcal{H}$ with coordinates $\mathbf{q}=\left(q_{2}, \ldots, q_{5}\right)$, for which $q_{2}^{2}+q_{3}^{2}+q_{4}^{2}+q_{5}^{2}=\Delta_{\mathrm{Q}}^{2}$ is minimal. This side condition seems to be legitimate under the point of view that all $\Delta_{i}$ should be as small as possible, because of $0 \leq\left|q_{i}\right| \leq \frac{3}{2} \Delta_{\mathrm{L}}$. This relation can be proved as follows: Without loss of generality, we assume that $\mathrm{L}$ is located in the positive sector, to say, $l_{2}=\cdots=l_{5}=\Delta_{\mathrm{L}}$. Due to Thales' theorem, the footpoints $\mathbf{F}$ with $\mathbf{f}=\left(f_{2}, f_{3}, f_{4}, f_{5}\right)$ of the perpendiculars drawn from the origin to all possible hyperplanes through $L$ are lying on the 
hypersphere

$$
\Lambda: \quad \sum_{i=2}^{5}\left(f_{i}-\frac{\Delta_{\mathrm{L}}}{2}\right)^{2}=\Delta_{\mathrm{L}}^{2}
$$

The maximum of $f_{i}$ is: $f_{i}=\frac{3}{2} \Delta_{\mathrm{L}}$ when $f_{j}=\frac{\Delta_{\mathrm{L}}}{2}$ for $j \in\{2,3,4,5\} \backslash\{i\}$.

$\mathrm{Q}$ is the point of contact between $\mathcal{H}$ and the hypershere $\Gamma$, centered in $\mathcal{K}_{o}$ (see figure 1), with

$$
q_{i}=\frac{-\operatorname{det}(\mathbf{J})}{\sum_{j=2}^{5}\left(\frac{\partial \operatorname{det}(\mathbf{J})}{\partial \theta_{j}}\right)^{2}} \cdot \frac{\partial \operatorname{det}(\mathbf{J})}{\partial \theta_{i}} \quad \Longrightarrow \quad \Delta_{\mathbf{Q}}=\frac{|\operatorname{det}(\mathbf{J})|}{+\sqrt{\sum_{j=2}^{5}\left(\frac{\partial \operatorname{det}(\mathbf{J})}{\partial \theta_{j}}\right)^{2}}}
$$

Theorem $7 \quad D M_{2}(\mathcal{K})$ approximately equals the shortest time $\Delta_{Q}(27)$ which is required to reach the next singularity, provided the sum of the squared angular velocities is bounded by 1. The following relationship holds:

$$
\Delta_{\mathrm{L}} \leq \Delta_{\mathrm{Q}} \leq 2 \Delta_{\mathrm{L}}
$$

Proof of the inequality: We have $\Delta_{\mathrm{L}}=\Delta_{\mathrm{Q}}$ when $\mathcal{H}$ touches the hypercube along a hyperplane. And for $L=Q$ the upper bound is reached.

By this method we obtain not only the desired distance measure, but also the worst instantaneous angular velocity ratio $\boldsymbol{\omega}_{-}:=\mathbb{R}^{+}\left(q_{2}, q_{3}, q_{4}, q_{5}\right)$ i.e., the direction towards the closest singularity. ${ }^{3}$ We can even evaluate any instantaneous angular velocity ratio $\boldsymbol{\omega}_{o}$ at the given non-singular posture $\mathcal{K}_{o}$. An adequate measure for this seems to be the angle

$$
\alpha:=\varangle\left(\boldsymbol{\omega}_{-}, \boldsymbol{\omega}_{o}\right)-\frac{\pi}{2} \in\left[-\frac{\pi}{2}, \frac{\pi}{2}\right] \quad \text { with } \quad \varangle\left(\boldsymbol{\omega}_{-}, \boldsymbol{\omega}_{o}\right) \in[0, \pi] .
$$

For $\alpha=-\frac{\pi}{2}$, one is moving directly to the singularity which is approximated by $\mathcal{H}$. $\alpha=\frac{\pi}{2}$ defines the best direction to avoid the singularity, and for $\alpha=0$ one is moving equidistantly to it.

\subsection{Closer inspection of $D M_{\infty}$ and $D M_{2}$}

For a better understanding of $D M_{\infty}$ and $D M_{2}$ we visualize the relationship between manipulability, $\Delta_{\mathrm{L}}, \Delta_{\mathrm{Q}}$ and $\boldsymbol{\omega}_{-}$. This will also help us to interpret the special cases of these two distance measures.

$\overline{3}$ With the method of 2.5.1 we were only able to give evidence about the worst instantaneous rotational direction of the joints by the vector $\left(\operatorname{sgn}\left(l_{2}\right), \operatorname{sgn}\left(l_{3}\right), \operatorname{sgn}\left(l_{4}\right)\right.$, $\left.\operatorname{sgn}\left(l_{5}\right)\right)$. 


\subsubsection{Vizualisation}

We look at the graph of the function $M P B(\mathcal{K}):=|\operatorname{det}(\mathbf{J})|$ over the space $\mathbb{V}_{\mathcal{K}}$, i.e., the map:

$$
\iota: \quad\left(\theta_{2}, . ., \theta_{5}\right) \mapsto\left(\theta_{2}, . ., \theta_{5},|\operatorname{det}(\mathbf{J})|\right) .
$$

The normal vector $\mathbf{n}_{o}$ at each point $\mathcal{K}_{o}$ of the hypersurface $\iota\left(\mathbb{V}_{\mathcal{K}}\right)$ is given by

$$
\mathbf{n}_{o}=\left(n_{2}, n_{3}, n_{4}, n_{5}, 1\right) \quad \text { with } \quad n_{i}:=-\operatorname{sgn}[\operatorname{det}(\mathbf{J})] \frac{\partial \operatorname{det}(\mathbf{J})}{\partial \theta_{i}} .
$$

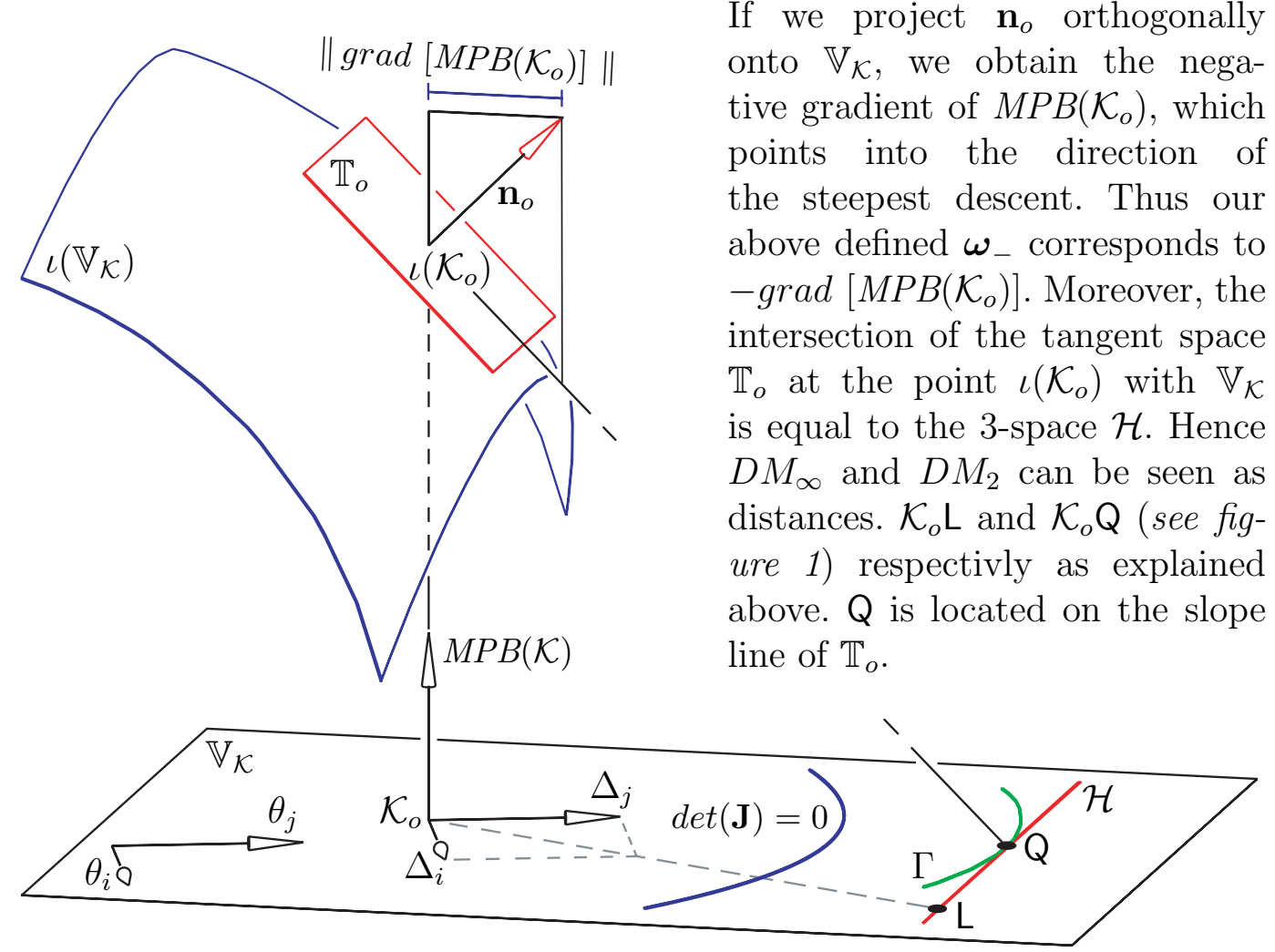

Fig. 1. The graph of $|\operatorname{det}(\mathbf{J})|$ over $\mathbb{V}_{\mathcal{K}}$

\subsubsection{Special cases and well-definedness}

(a) How can we interpret equation (24), if all coefficients of $\Delta_{i}(i=2, . ., 5)$ are equal to zero and $\operatorname{det}(\mathbf{J})=0$ ? In such a case the robot is in a singular posture of higher order, because all four partial derivatives vanish. Then $D M_{\infty}(\mathcal{K})=D M_{2}(\mathcal{K})=0(\Leftrightarrow \mathbf{l}=\mathbf{q}=\mathbf{o})$ as (24) holds for any $\left(\Delta_{2}, \ldots, \Delta_{5}\right) \in \mathbb{R}^{4}$.

(b) A problem occurs if the four partial derivatives of the Jacobian's determinant vanish but $\operatorname{det}(\mathbf{J}) \neq 0$. Then equation (24) can never be fulfilled. 
Every $6 \mathrm{R}$ manipulator has at least such a posture, because the four unknowns $\theta_{2}, \ldots, \theta_{5}$ have to fulfill four equations, namely the four partial derivatives are simultaneously equal to zero.

In this case $\operatorname{grad}\left[M P B\left(\mathcal{K}_{o}\right)\right]=\mathbf{o}$ and so $\mathbf{n}_{o} \perp \mathbb{V}_{\mathcal{K}}$, which is equivalent to $\mathbb{T}_{o} \| \mathbb{V}_{\mathcal{K}}$. Consequently, $\mathcal{H}$ is the ideal hyperplane of $\mathbb{V}_{\mathcal{K}} . \mathcal{K}_{o}$ has the maximal distance $D M_{\infty}\left(\mathcal{K}_{o}\right)=D M_{2}\left(\mathcal{K}_{o}\right) \rightarrow \infty$, because $\mathrm{Q}$ and $\mathrm{L}$ are ideal points.

In cases $(\mathbf{a})$ and (b) the special configuration $\mathcal{K}_{o}$ is a stationary point of the vector field $-\operatorname{grad}\left[M P B\left(\mathcal{K}_{o}\right)\right]$. As a consequence $\alpha$ is not defined in case (b). In order to evaluate $\boldsymbol{\omega}_{o}$ of $\mathcal{K}_{o}$, we have to look at the derivatives of order 2 , thus the curvature of $\iota\left(\mathbb{V}_{\mathcal{K}}\right)$ at $\iota\left(\mathcal{K}_{o}\right)$. So we can define $\boldsymbol{\omega}_{-}$in $\mathcal{K}_{o}$ by the direction $\boldsymbol{\kappa}$ of the principal curvature with the smallest absolute value:

$$
\boldsymbol{\omega}_{-}:= \pm \boldsymbol{\kappa} \quad \Longrightarrow \quad \varangle\left(\boldsymbol{\omega}_{-}, \boldsymbol{\omega}_{o}\right) \in\left[0, \frac{\pi}{2}\right] \quad \Longrightarrow \quad \alpha \in\left[-\frac{\pi}{2}, 0\right] .
$$

Theorem $8 \quad D M_{\infty}$ and $D M_{2}$ have all six properties required in section 1.

Proof: $D M_{\infty}$ and $D M_{2}$ trivially fulfill the properties 1 and 2, and also the fifth, because of Theorem 6 and Theorem 7 . Now we look at equation (24) to show the remaining properties: The equation's coefficients $\operatorname{det}\left(\mathbf{J}_{i, j}\right)$ are for the same reasons as the Jacobian's determinant invariant with respect to Euclidean motions (see (20)). Furthermore this equation is invariant under similarities, because if we change the scaling by the factor $\lambda$ the whole equation is multiplied by $\lambda^{3}$.

Due to the approximation of direct kinematics, $\Delta_{\mathrm{L}}$ and $\Delta_{\mathrm{Q}}$ are computable in real time, which is an important criterion for practical applications.

\section{Final Example}

We take the industrial robot $A B B$ IRB 2000 as a particular example (see figure 2). We look at the constrained motion which is determined by:

$\theta_{1}=\theta_{6}=0, \quad \theta_{2}=t \frac{21 \pi}{18}-\frac{11 \pi}{18}, \quad \theta_{3}=t^{2} \frac{2 \pi}{3}-\frac{\pi}{3}, \quad \theta_{4}=t \pi-\frac{\pi}{2}, \quad \theta_{5}=t \frac{4 \pi}{3}-\frac{2 \pi}{3}$.

For $t \in\left[t_{1}, t_{2}\right]$ the graphs of the EE independent performance indices and of $\alpha$ are displayed in figure 3 , where $\mathcal{K}\left(t_{1}\right)$ and $\mathcal{K}\left(t_{2}\right)$ are singular configurations. $D M_{\infty}(\mathcal{K})$ and $D M_{2}(\mathcal{K})$ indicate the closeness to the next singularity at least as good as $M P B(\mathcal{K})$, but these indices have the big advantage of being invariant under similarities and of having a geometric meaning for the $6 \mathrm{R}$ robot.

We assume that the operation ellipsoid required for $C D N_{O E}$ and $P I_{O E}$ is a sphere of radius $R$ centered in $O P$ which has the coordinates $(835,0,1150)$ in the initial position. The counters of $P I_{O E}$ resp. $C D N_{O E}^{-1}$ over $R \times t \in[50,300] \times$ 
$\left[t_{1}, t_{2}\right]$ are displayed in figure 4 resp. figure 5. As it can be seen in figure 5, the index $C D N_{O E}$ depends strongly on $R$. Therefore it is very important to choose for each application of the robot the right operation ellipsoid if taking $C D N_{O E}$ for robot control. As already said, the operation sphere with radius $C L \sqrt{3 / 2}$ generally does not fit the part of the EE which one is interested to manipulate. Because of the strong dependency of $C D N_{O E}$ on $R$ one cannot expect the same for $P I_{O E}$. $P I_{O E}$ is more or less constant for $R \in[50,300]$ (see figure 4). It should be said that this is not the general case, but this example shows very well the different behaviour of these two close related indices.

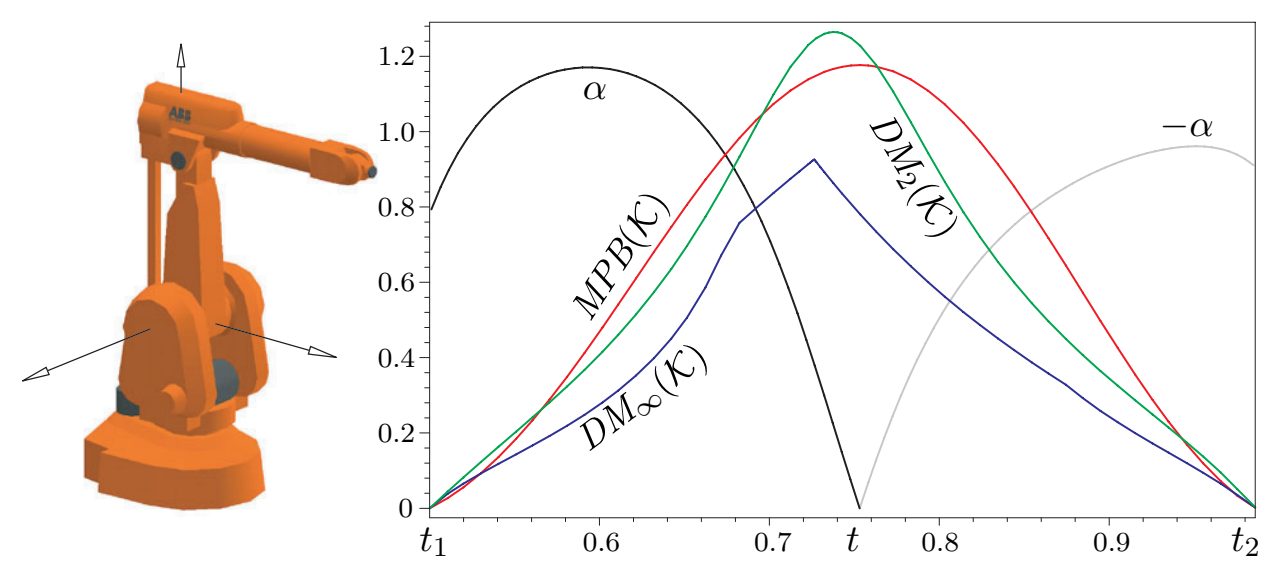

Fig. 2. Initial position

Fig. 3. Graphs of $M P B(\mathcal{K}), D M_{\infty}(\mathcal{K}), D M_{2}(\mathcal{K}), \alpha$ of the IRB 2000 robot

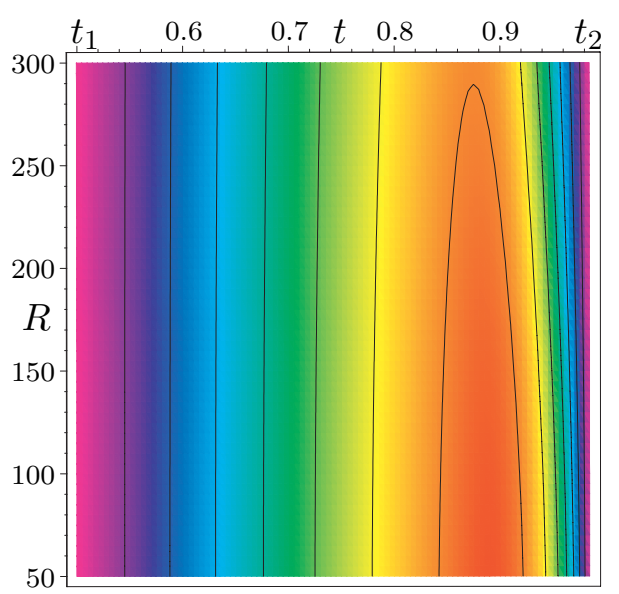

Fig. 4. Counters of $P I_{O E}$

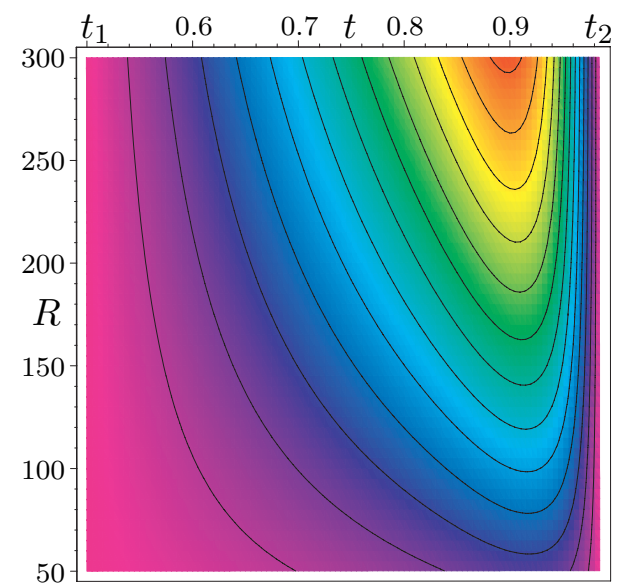

Fig. 5. Counters of $C D N_{O E}^{-1}$

\section{Conclusion}

In this paper we presented four new performance indices for control which have all six properties required in section 1 . Two of them depend on the 
end-effector and the other two do not. The EE dependent indices $P I_{O E}$ and $C D N_{O E}$ are based on the operation ellipsoid and on an object-oriented metric in the workspace. We proved that $C D N_{C L}$ can be seen as a special case of $C D N_{O E}$. Therefore it was possible to give a geometric interpretation of the characteristic length.

The EE independent indices $D M_{\infty}$ and $D M_{2}$ reflect the distance of the actual posture $\mathcal{K}$ from the closest singularity. These distance measures take the possible variation of the joint axes into account, because they are based on a linearized approximation of direct kinematics.

\section{Acknowledgment}

This is part of the author's PhD-thesis. The author expresses his sincere thanks to his supervisor Prof. H. Stachel for continuous support. The author would also like to thank the anonymous reviewers, who have helped to improve the quality of this paper.

\section{References}

[1] Angeles, J., Fundamentals of Robotic Mechanical Systems. Theory, Methods, and Algorithms. Springer, $2^{\text {nd }}$ Edition, 2002.

[2] Angeles, J. and Lopez-Cajun, C.S., Kinematic Isotropy and the Conditioning Index of Serial Robotic Manipulators. Int. J. of Robotics Research 11 (6) (1992) 560-571.

[3] Gosselin, C.M., Dexterity Indices for Planar and Spatial Robotic Manipulators. IEEE Int. Conf. on Robotics and Automation 1 (1990) 650-655.

[4] Hofer, M., Pottmann, H., and Ravani, B., From curve design algorithms to the design of rigid body motions. The Visual Computer 20 (5) (2004) 279-297.

[5] Nawratil, G., The Control Number as Index for Stewart Gough Platforms. Advances in Robot Kinematics: Mechanisms and Motion (J. Lenarcic, B. Roth eds.), Springer, 2006, pp. 15-22.

[6] Nawratil, G., Neue Kinematische Performance-Indizes für 6R Roboter und Stewart Gough Plattformen. PhD-thesis, Vienna University of Technology, in preperation.

[7] Salisbury, J.K. and Craig, J.J., Articulated Hands: Force Control and Kinematic Issues. Int. J. of Robotics Research 1 (1) (1982) 4-17.

[8] Stachel, H., Instantaneous spatial kinematics and the invariants of the axodes. Proc. Ball 2000 Symposium, Cambridge, no. 23. 
[9] Tandirci, M., Angeles, J., and Ranjbaran, F., The Characteristic Point and the Characteristic Length of Robotic Manipulators. Proc. ASME $22^{\text {nd }}$ Biennial Conf. Robotics, Spatial Mechanisms, and Mechanical Systems 45 (1992) 203208.

[10] Yoshikawa, T., Manipulability of Robotic Mechanisms. Int. J. of Robotics Research 4 (2) (1985) 3-9. 